\title{
Retinoic acid induces macrophage cholesterol efflux and inhibits atherosclerotic plaque formation in apoE-deficient mice
}

\author{
Wenjing Zhou ${ }^{1,2} \dagger$, Jiacheng Lin $^{3} \dagger$, Hongen Chen ${ }^{1,2}$, Jingjing Wang ${ }^{1,2}$, Yan Liu ${ }^{1,2}$ and Min Xia ${ }^{1,2 *}$ \\ ${ }^{1}$ Guangdong Provincial Key Laboratory of Food, Nutrition and Health, School of Public Health, Sun Yat-sen University \\ (Northern Campus), Guangzhou, Guangdong Province 510080, People's Republic of China \\ ${ }^{2}$ Department of Nutrition, School of Public Health, Sun Yat-sen University (Northern Campus), Guangzhou, Guangdong \\ Province 510080, People's Republic of China \\ ${ }^{3}$ Guanghua School of Stomatology, Guangdong Provincial Key Laboratory of Stomatology, Sun Yat-sen University, \\ Guangzhou, Guangdong Province 510055, People's Republic of China
}

(Submitted 23 December 2014 - Final revision received 18 May 2015 - Accepted 22 May 2015 - First published online 23 July 2015)

Abstract

It has been suggested that retinoic acid (RA) has a potential role in the prevention of atherosclerotic CVD. In the present study, we used J774A.1 cell lines and primary peritoneal macrophages to investigate the protective effects of RA on foam cell formation and atherogenesis in apoE-deficient $\left(\mathrm{apoE}^{-/-}\right)$mice. A total of twenty male apoE ${ }^{-/-}$mice ( $n 10$ animals per group), aged 8 weeks, were fed on a high-fat diet (HFD) and treated with vehicle or 9-cis-RA for 8 weeks. The atherosclerotic plaque area in the aortic sinus of mice in the 9-cis-RA group was $40.7 \%$ less than that of mice in the control group $(P<0.01)$. Mouse peritoneal macrophages from the 9-cis-RA group had higher protein expression levels of ATP-binding cassette transporter A1 (ABCA1) and G1 (ABCG1) than those from the control group. Serum total and LDL-cholesterol concentrations were lower in the 9-cis-RA group than in the control group $(P<0.05)$. In vitro studies showed that incubation of cholesterol-loaded J774A.1 macrophages with 9-cis-RA $(0 \cdot 1,1$ and $10 \mu$ mol/1) induced cholesterol efflux in a dose-dependent manner. The 9-cis-RA treatment markedly attenuated lipid accumulation in macrophages exposed to oxidised LDL. Moreover, treatment with 9-cis-RA significantly increased the protein expression levels of ABCA1 and ABCG1 in J774A.1 macrophages in a dose-dependent manner. Furthermore, 9-cis-RA dose-dependently enhanced the protein expression level of liver X receptor- $\alpha$ $(\mathrm{LXR} \alpha)$, the upstream regulator of ABCA1 and ABCG1. Taken together, the present results show that 9-cis-RA suppresses foam cell formation and prevents HFD-induced atherogenesis via the LXR $\alpha$-dependent up-regulation of ABCA1 and ABCG1.

Key words: Retinoic acid: Macrophage cholesterol efflux: ApoE-deficient mice: Atherosclerosis

Atherosclerotic CVD have been the leading cause of death in Western societies. Accumulation of macrophage-derived foam cells is the hallmark of early-stage atherosclerosis ${ }^{(1,2)}$. The formation of macrophage foam cells is mainly due to the uncontrolled uptake of modified LDL by macrophages, resulting in excessive lipoprotein-derived cholesterol accumulation inside the cells. Clearance of arterial cholesterol deposits by macrophages is beneficial during the early stages of atherogenesis ${ }^{(3)}$.

Cholesterol efflux from macrophages is the first and potentially the most important step in reverse cholesterol transport, a process especially relevant to atherosclerosis and to the regression of atherosclerotic plaques ${ }^{(4,5)}$. The ATP-binding cassette transporter family, including ABCA1 and ABCG1, is highly expressed in lipid-loaded macrophages and mediates cholesterol efflux ${ }^{(6-8)}$. ABCA1 is a key regulator of cholesterol and phospholipid export to lipid-free apolipoproteins, forming nascent $\mathrm{HDL}^{(9)}$. ABCG1 was identified as a more likely mediator of cholesterol transport to HDL ${ }^{(10)}$. Both ABCA1 and ABCG1 have been reported to be mediated by liver X receptors $(\mathrm{LXR})^{(11,12)}$, the key regulators of lipid and cholesterol metabolism.

The effects of vitamin A on growth and development are achieved through the action of biologically active metabolites, retinoic acids (RA), mainly including all-trans-RA and 9-cis-retinoic acid (9-cis-RA) ${ }^{(13,14)}$. RA is synthesised primarily intracellularly from retinaldehyde, which itself can be produced from retinol or provitamin A carotenoids such as

\footnotetext{
Abbreviations: 9-cis-RA, 9-cis-retinoic acid; ABCA1, ATP-binding cassette transporter A1; ABCG1, ATP-binding cassette transporter G1; FXR, farnesoid $\mathrm{X}$ receptor; LXR, liver $\mathrm{X}$ receptor; LXRE, liver $\mathrm{X}$ receptor response element; MPM, mouse peritoneal macrophages; PXR, pregnane $\mathrm{X}$ receptor; RA, retinoic acid; RPMI, Roswell Park Memorial Institute; RXR, retinoid X receptor; siRNA, small interfering RNA.
}

*Corresponding author: Professor M. Xia, fax +86 20 87330446, email xiamin@mail.sysu.edu.cn

† Both authors contributed equally to this work. 
$\beta$-carotene ${ }^{(15)}$. The broad range of biological effects exerted by RA is mainly attributable to the control of specific gene expression. RA binds to and activates the members of the nuclear receptor family including retinoic acid receptor and retinoid X receptor (RXR), which then form heterodimers with potential partners and bind to the response element in the promoter of target genes to initiate gene transcription $^{(16-18)}$. LXR, farnesoid X receptor (FXR) and pregnane X receptor (PXR) are three major potential partners of RXR, which modulate systematic lipid metabolism. Recent studies have confirmed the regulatory role of RA in lipid metabolism in various cell types including macrophages. RA effectively regulates the expression of ABCA1 in many macrophage cell types ${ }^{(19)}$. All-trans-RA increased the protein expression levels of ABCG1 and cholesterol efflux from the macrophages ${ }^{(20)}$. 9-cis-RA has also been reported to induce ABCA1 expression and cholesterol efflux in brain cells ${ }^{(21)}$. Although the critical role of RA in cholesterol efflux in vitro has been fully demonstrated, the impact of 9-cis-RA on macrophage foam cell formation and atherosclerosis in vivo and the underlying molecular mechanisms are still obscure, and need to be further explored.

The purpose of the present study was to investigate the protective effects of 9-cis-RA on foam cell formation and atherosclerosis in $\mathrm{apoE}^{-1-}$ mice, and to uncover the underlying molecular mechanism.

\section{Materials and methods}

\section{Materials}

9-cis-RA was obtained from Enzo Life Sciences. apoAI was obtained from human plasma and other reagents were purchased from Sigma-Aldrich, unless otherwise indicated. liver X receptor response element (LXRE)-Luc and farnesoid $\mathrm{X}$ receptor response element (FXRE)-Luc reporter plasmids were purchased from Genomeditech. Pregnane X receptor response element (PXRE)-Luc reporter plasmid was constructed by cloning PXR binding sequences in the promoter area of ABCA1 and ABCG1 into the vector pEZX-PG02 upstream of the firefly luciferase reporter gene (GeneCopoeia).

\section{Animal experiments}

Male homozygous apoE ${ }^{-/-}$mice $(\mathrm{C} 57 \mathrm{BL} / 6$ genetic background), aged 8 weeks, were obtained from Jackson Laboratories and randomly divided into two groups ( $n 10$ animals per group). Mice were fed on a Western-type diet (containing $0 \cdot 15 \%$ cholesterol and $41 \%$ saturated fat, MD12015; Medicience Limited; Table 1) for 8 weeks. 9-cis-RA was dissolved in dimethyl sulfoxide, and after dilution with PBS containing $0.5 \%$ bovine serum albumin, a dose of $2 \mathrm{mg} / \mathrm{kg}$ was administered intraperitoneally four to five times per week (9-cis-RA group). PBS containing $0.5 \%$ bovine serum albumin was administered as vehicle to the control group. Body weight and food intake were recorded weekly. At the end of the experiment, mice were killed by $\mathrm{CO}_{2}$ inhalation and serum and tissue samples were collected and stored at
Table 1. Composition of the experimental diet

\begin{tabular}{lc}
\hline Nutrients & High-fat diet (MD12015) \\
\hline Energy & \\
$\mathrm{kcal} / \mathrm{g}$ & $4 \cdot 7$ \\
$\mathrm{~kJ} / \mathrm{g}$ & $19 \cdot 7$ \\
Ingredients (g/kg) & \\
Casein & $195 \cdot 0$ \\
Maltodextrin & $60 \cdot 0$ \\
Sucrose & $340 \cdot 0$ \\
Anhydrous milk fat & $210 \cdot 0$ \\
Soyabean oil & $20 \cdot 0$ \\
Starch & $55 \cdot 46$ \\
Cellulose & $50 \cdot 0$ \\
Mineral mix & $43 \cdot 0$ \\
Vitamin mix* & $19 \cdot 0$ \\
L-Cys & $3 \cdot 0$ \\
Choline bitartrate & $3 \cdot 0$ \\
Cholesterol & 1.5 \\
TBHQ, antioxidant & 0.04 \\
Total (g) & 1000 \\
\hline
\end{tabular}

TBHQ, tertiary butylhydroquinone.

*From Reeves et al. ${ }^{(49)}$

$-80^{\circ} \mathrm{C}$ until analysis. All animal experiments were approved by the Institutional Animal Care and Use Committee of Sun Yat-sen University.

\section{Measurement of serum lipid concentrations}

Serum samples were collected at the end of the experiment. After $12 \mathrm{~h}$ fasting, venous blood was collected from the lateral saphenous vein into Eppendorf tubes. Serum was isolated by centrifugation at $2800 \mathrm{~g}$ for $20 \mathrm{~min}$ at $4^{\circ} \mathrm{C}$, and stored at $-80^{\circ} \mathrm{C}$ until analysis. Enzymatic colorimetric methods with diagnostic reagents from Wako on an Automatic Biochemistry Analyzer (Hitachi 7600-010) were used to determine total cholesterol (Cholesterol E, catalogue no. 439-17 501), LDL-cholesterol (L-Type LDL-C, catalogue no. 993-00 404), HDL-cholesterol (L-Type HDL-C, catalogue no. 997-72591) and TAG (L-Type TAG M, catalogue no. 461-08992).

\section{Measurement of serum total retinoic acid concentrations}

Serum RA concentration was measured using a commercially available ELISA kit (Biosamite), according to the manufacturer's protocol.

\section{Evaluation of atherosclerotic lesions}

Mice were perfused with $10 \%$ buffered formalin, and the heart was then dissected out. The base of each heart was frozen in O.C.T. tissue-freezing medium (Leica) and cryosectioned, as described previously ${ }^{(22)}$. For quantification of the maximum lesion area within the aortic sinus, the cross-sectional area of Oil red $O$ staining was measured from the points of emergence to the points of disappearance of the aortic valves.

\section{Isolation of primary mouse macrophages}

Mouse peritoneal macrophages (MPM) from the two experimental groups were isolated $3 \mathrm{~d}$ after intraperitoneal injection 
with $4 \%$ thioglycolate solution, as described previously ${ }^{(23)}$. After massage of the abdomen, MPM were collected and then centrifuged at $2000 \mathrm{rpm}$ for $5 \mathrm{~min}$, and resuspended in serum-free Roswell Park Memorial Institute (RPMI)-1640 medium at $37^{\circ} \mathrm{C}$ in a humidified atmosphere of $5 \% \mathrm{CO}_{2}$ for at least $2 \mathrm{~h}$. Then, cells were washed with PBS three times to remove non-adherent cells.

\section{Cell culture and treatments}

The J774A.1 cell line was obtained from the American Type Culture Collection. Cells were seeded in six-well plates at a density of $1.0 \times 10^{6}$ cells per well in RPMI-1640 medium (Gibco; Life Technologies) containing 10\% fetal bovine serum, and were maintained at $37^{\circ} \mathrm{C}$ in a humidified atmosphere of $5 \% \mathrm{CO}_{2}$. Cells were treated with vehicle (dimethyl sulfoxide), 9-cis-RA (0.1, 1 and $10 \mu \mathrm{mol} / \mathrm{l})$ or $Z$-guggulsterone $(100 \mu \mathrm{mol} / \mathrm{l})$ for $24 \mathrm{~h}$ before analysis.

\section{Cholesterol efflux assay}

J774A.1 cells were equilibrated with 12-[(7-nitro-2,1,3-benzoxadiazol-4-yl)amino]-(3S,10R,13R)-3-methoxy-10,13-dimethyl-17((R)-6-methylheptan-2-yl)-tetradecahydro-1H-cyclopenta[ $\alpha]$ phenanthrene (NBD-cholesterol) $(1 \mu \mathrm{g} / \mathrm{ml}$; Cayman Chemical) for 6h. NBD-cholesterol-labelled cells were washed with PBS and incubated in serum-free RPMI-1640 medium containing apoAI $(10 \mu \mathrm{g} / \mathrm{ml}$; Calbiochem) or HDL $(50 \mu \mathrm{g} / \mathrm{ml}$; Calbiochem) for another $6 \mathrm{~h}$. The fluorescence-labelled cholesterol released from the cells into the medium was measured with a microplate reader (Tecan Infinite F200). Cholesterol efflux was expressed as a percentage of fluorescence in the medium relative to the total amounts of fluorescence detected in the cells and the medium. Each experiment was performed in triplicate for at least three times ${ }^{(24)}$.

\section{Confocal microscopy analysis}

Cells were fixed with $4 \%$ paraformaldehyde for $15 \mathrm{~min}$ at room temperature. Then, cells were incubated with the fluorescence probe BODIPY ${ }^{\circledR}$ 493/503 (4,4-difluoro-1,3,5,7,8-pentamethyl-4-bora-3a,4a-diaza-s-indacene, $1 \mathrm{mg} / \mathrm{ml}$; Invitrogen) for $1 \mathrm{~h}$ in the dark, followed by incubation with the nuclei staining 4',6-diamidino-2-phenylindole (DAPI, $5 \mu \mathrm{g} / \mathrm{ml}$; Beyotime) at $37^{\circ} \mathrm{C}$ for another $30 \mathrm{~min}$. Images were captured using the laser scanning confocal microscope (Leica TCS SP5 II). The 488-line argon laser was used to capture BODIPY ${ }^{\circledR} 493 / 503$ lipid staining, and the $405 \mathrm{~nm}$ laser diode was used to capture the DAPI nuclear stain. Intracellular fluorescence intensity was quantified by Image software (National Institutes of Health) ${ }^{(25)}$.

\section{Quantitative real-time RT-PCR}

Total RNA was isolated using TRIzol Reagent (Invitrogen). Complementary DNA was synthesised from total RNA by RT-PCR (Takara). The resulting complementary DNA was subjected to real-time RT-PCR analysis with gene-specific primers in the presence of the SYBR Green PCR master mix in an ABI Prism VR7 instrument (Applied Biosystems).
The primer sequences used were as follows: ABCA1 - forward primer 5'-AAA ACC GCA GAC ATC CTT CAG-3', reverse primer 5'-CAT ACC GAA ACT CGT TCA CCC- $3^{\prime}$; ABCG1 - forward primer 5'-CTT TCC TAC TCT GTA CCC GAG G-3', reverse primer 5'-CGG GGC ATT CCA TTG ATA AGG- $3^{\prime}$; LXR $\alpha$ - forward primer 5'-CTC AAT GCC TGA TGT TTC TCC T-3', reverse primer 5'-TCC AAC CCT ATC CCT AAA GCA A- $3^{\prime}$; LXR $\beta$ - forward primer 5'-ATG TCT TCC CCC ACA AGT TCT-3', reverse primer 5'-GAC CAC GAT GTA GGC AGA GC-3'; FXR - forward primer 5'-GCT TGA TGT GCT ACA AAA GCT G-3', reverse primer 5'-CGT GGT GAT GGT TGA ATG TCC-3'; PXR - forward primer 5'-TAG GGA CCT GCC TAT TGA GGA-3', reverse primer 5'-CCG TTT CCG TGT CGA ACA TC-3'; glyceraldehyde 3-phosphate dehydrogenase - forward primer 5'-CCA CAG CTG AGA GGG AAA TC-3', reverse primer 5'-AAG GAA GGC TGG AAA AGA GC- $3^{\prime}$. Data were analysed using the $2^{-\Delta \Delta C_{\mathrm{T}}}$ threshold cycle method. Glyceraldehyde 3-phosphate dehydrogenase was used as an invariant internal control.

\section{Western blot}

Cells were harvested and protein extracts were prepared as described previously ${ }^{(22)}$. Aliquots $(40 \mu \mathrm{g})$ of protein extracts were separated by SDS-PAGE and then transferred onto an Immobilon-P transfer membrane (Millipore). After blocking, the blots were incubated with various primary antibodies followed by horseradish peroxidase-labelled secondary antibodies. Protein bands were detected with an ECL Western Blotting Substrate Kit (Pierce). The primary antibodies used were as follows: anti-ABCA1 (Novus); anti-ABCG1 (Novus); anti-LXR $\alpha / \beta$ (Santa Cruz Biotechnology); anti-FXR (Bioss); anti-PXR (Proteintech); anti-glyceraldehyde 3-phosphate dehydrogenase (Cell Signaling Technology).

\section{Luciferase assay}

The 293T cell line was obtained from the American Type Culture Collection. Cells were grown in Dulbecco's modified Eagle's medium (Gibco; Life Technologies) containing 10\% fetal bovine serum and were maintained at $37^{\circ} \mathrm{C}$ in a humidified atmosphere of $5 \% \mathrm{CO}_{2}$. Cells were seeded in forty-eightwell plates and transiently co-transfected with LXRE/FXRE/ PXRE-Luc reporter plasmid $(0.4 \mu \mathrm{g})$ and pRL-SV40 Vector $(0.02 \mu \mathrm{g})$ with Lipofectamine 2000 (Invitrogen), according to the manufacturer's instructions. After transfection for $24 \mathrm{~h}$, cells were treated with vehicle (dimethyl sulfoxide) or 9-cisRA $(0 \cdot 1,1$ and $10 \mu \mathrm{mol} / \mathrm{l})$ for another $24 \mathrm{~h}$. Subsequently, the amount of Luciferase activity was measured and normalised to the amount of Renilla luciferase activity using the Dual-Luciferase Reporter Assay System (Promega).

\section{Liver $X$ receptor- $\alpha$ small interfering RNA transfection}

For the small interfering RNA (siRNA)-mediated downregulation of LXR $\alpha, \mathrm{LXR} \alpha$-specific siRNA and non-targeting control siRNA were purchased from Santa Cruz Biotechnology. For transfection with siRNA, J774A.1 cells were plated at a density of $2.0 \times 10^{6}$ cells per well. Cells were transfected with LXR $\alpha$ 
Table 2. Serum retinoic acid (RA) and lipid concentrations in apoEdeficient mice

(Mean values and standard deviations; $n$ 10)

\begin{tabular}{lccccc}
\hline & \multicolumn{2}{c}{ Control } & & \multicolumn{2}{c}{$9-$ cis-RA } \\
\cline { 2 - 3 } Variables & Mean & SD & & Mean & SD \\
\hline TC $(\mathrm{mmol} / \mathrm{l})$ & $18.31^{\mathrm{a}}$ & 2.77 & & $11.74^{\mathrm{b}}$ & 3.96 \\
TAG $(\mathrm{mmol} / \mathrm{l})$ & 1.10 & 0.35 & & 1.16 & 0.33 \\
HDL-C $(\mathrm{mmol} / \mathrm{l})$ & $0.56^{\mathrm{a}}$ & 0.09 & & $0.74^{\mathrm{b}}$ & 0.28 \\
LDL-C $(\mathrm{mmol} / \mathrm{l})$ & $3.95^{\mathrm{a}}$ & 0.76 & & $2.92^{\mathrm{b}}$ & 0.22 \\
RA $(\mathrm{nmol} / \mathrm{l})$ & $--^{\mathrm{a} *}$ & - & & $339^{\mathrm{b}}$ & 34.19 \\
\hline
\end{tabular}

TC, total cholesterol; HDL-C, HDL-cholesterol; LDL-C, LDL-cholesterol.

${ }^{a, b}$ Mean values within a row with unlike superscript letters were significantly different $(P<0.05)$.

* Serum 9-cis-RA concentration for the control group was below the detection limit.

siRNA at a concentration of $100 \mathrm{nmol} / 1$ for $48 \mathrm{~h}$ followed by treatment with 9-cis-RA $(10 \mu \mathrm{mol} / \mathrm{l})$ for an additional $24 \mathrm{~h}$.

\section{Statistical analysis}

All experiments were carried out for at least three times. Data are presented as means and standard deviations. Statistical analysis was performed using SPSS (version 17.0, IBM SPSS Statistics; IBM Corporation). Significance was determined by two-tailed Student's $t$ test or ANOVA. $P<0.05$ was considered as statistically significant.

\section{Results}

\section{Body weight and food intake}

Body weight did not differ between the two groups at baseline (18.5 (SD 1.74) $\mathrm{g}$ for the control group and 17.8 (SD 2.91) $\mathrm{g}$ for the RA group) or at the end of the experiment $(31.23$ (SD 3.56) $\mathrm{g}$ for the control group and 28.50 (SD 2.90) g for the RA group). There was no difference in daily food intake between the control group $(2.50(\mathrm{SD} 0.07) \mathrm{g} / \mathrm{d})$ and the RA group $(2.55(\mathrm{SD} 0.07) \mathrm{g} / \mathrm{d})$. The amount of RA injected was $0.05(\mathrm{SD} 0.01) \mathrm{mg} / \mathrm{d}$ per mouse.

\section{Serum retinoic acid and lipid concentrations}

The serum concentration of RA was $<10 \mathrm{nmol} / \mathrm{l}$ (assay sensitivity $10 \mathrm{nmol} / \mathrm{l}$ ) for the control group and 339 (SD 34.19) nmol/1 for the RA group (Table 2).
Serum total and LDL-cholesterol concentrations were lower in the RA group than in the control group $(P<0 \cdot 05)$. Serum HDL-cholesterol concentrations were higher in the RA group than in the control group $(P<0 \cdot 05)$. Serum TAG concentrations did not differ between the two groups (Table 2 ).

\section{Atherosclerotic plaque area}

The mean atherosclerotic lesion area in the aortic sinus of the RA group (29288.77 (SD 1472.92) $\mu^{2}$ ) was $40.7 \%$ less than that of the control group $\left(49397.86\right.$ (SD 1179.69) $\left.\mu \mathrm{m}^{2}\right)$ $(P<0 \cdot 01)$ (Fig. 1(a) and (b)).

\section{ATP-binding cassette transporter A1/ATP-binding cassette transporter $G 1$ expression and lipid accumulation in mouse peritoneal macrophages}

MPM isolated from apoE ${ }^{-/-}$mice in the RA group had higher protein expression levels of ABCA1, ABCG1 and LXR $\alpha$ compared with those in the control group (all $P<0.05$; Fig. 2(a) and (b)). The results of BODIPY ${ }^{\circledR} 493 / 503$ lipid staining showed that the lipid content in MPM from the RA group was significantly reduced compared with the control group $(P<0 \cdot 01$; Fig. 2(c) and (d)).

\section{Retinoic acid induces cholesterol efflux from macrophages}

Incubation of J774A.1 macrophages with increasing concentrations of 9-cis-RA dose-dependently increased NBD-cholesterol efflux to apoAI compared with the control treatment $(P<0.01$; Fig. 3(a)). Moreover, the 9-cis-RA treatment also dose-dependently promoted NBD-cholesterol efflux to HDL to a less extent compared with the control treatment $(P<0 \cdot 05$; Fig. 3(b)).

\section{Retinoic acid decreases intracellular lipid accumulation in macrophages}

Incubation of cholesterol-preloaded J774A.1 macrophages with 9-cis-RA significantly decreased intracellular lipid accumulation compared with the control treatment $(P<0 \cdot 01$; Fig. 4(a) and (b))

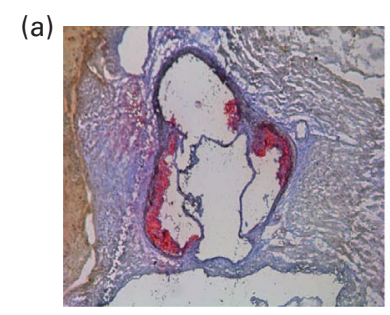

Control

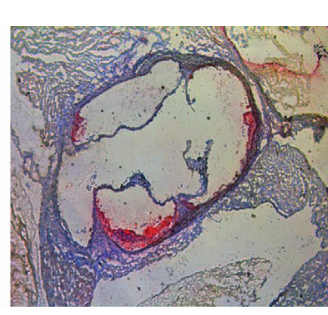

9-cis-RA

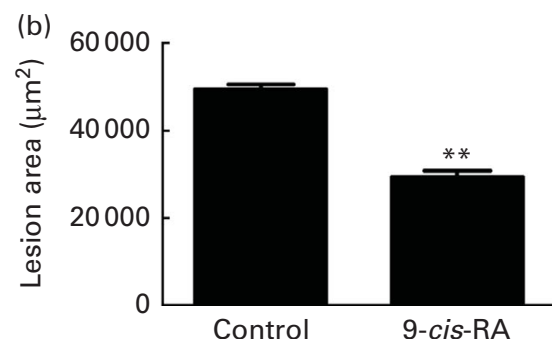

Fig. 1. Atherosclerotic lesions in the aortic sinus of apoE-deficient mice fed a high-fat diet for 8 weeks and treated with 9-cis-retinoic acid (9-cis-RA) or vehicle. (a) Representative photomicrographs of the aortic sinus stained with Oil red O. Magnification $400 \times$. (b) The lesion area in the aortic sinus was calculated using Leica software version 3.8. Values are means $(n 10)$, with their standard deviations represented by vertical bars. ${ }^{\star \star}$ Mean value was significantly different from that of the control group $(P<0.01)$. (A colour version of this figure can be found online at http://www.journals.cambridge.org/bjn). 
(a)

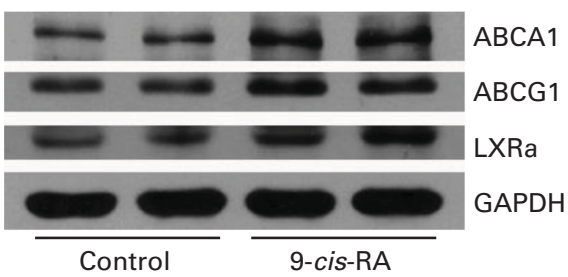

(c)

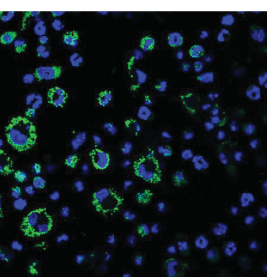

Control
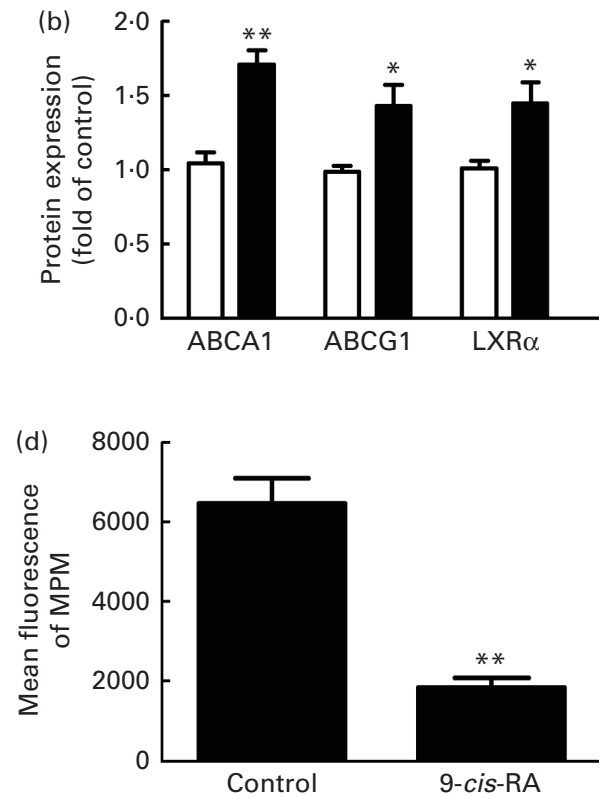

Fig. 2. ATP-binding cassette transporter $A 1$ (ABCA1)/ATP-binding cassette transporter G1 (ABCG1) expression and intracellular lipid accumulation. ApoE-deficient mice fed a high-fat diet were treated with 9-cis-retinoic acid (9-cis-RA; $\mathbf{0})$ or vehicle (control; $\square$ ) for 8 weeks. (a) Protein expression levels of ABCA1, ABCG1 and liver $X$ receptor- $\alpha(L X R \alpha)$ in mouse peritoneal macrophages (MPM). (b) Quantification of ABCA1, ABCG1 and LXR $\alpha$ protein density normalised to the expression level of glyceraldehyde 3-phosphate dehydrogenase (GAPDH), and expressed as optical density relative to that of the control. (c) Overlap images of BODIPY ${ }^{\circledR} 493 / 503$ and DAPI (4',6-diamidino-2-phenylindole) of MPM. Magnification 400×. (d) Mean fluorescence was calculated using ImageJ software. Values are means ( $n$ 10), with their standard deviations represented by vertical bars. Mean value was significantly different from that of the control group: ${ }^{*} P<0.05$, ${ }^{\star \star} P<0.01$. (A colour version of this figure can be found online at http://www.journals.cambridge.org/bjn).

\section{Retinoic acid increases ATP-binding cassette transporter A1 and ATP-binding cassette transporter G1 expression in macrophages}

Compared with the control treatment, 9-cis-RA-treated J774A.1 macrophages increased the mRNA expression levels of $A B C A 1, A B C G 1$ and $L X R \alpha$ in a dose-dependent manner $(P<0.05)$ (Fig. 4(c)). The treatment of J774A.1 macrophages with 9-cis-RA dose-dependently increased the protein expression levels of ABCA1, ABCG1 and $\mathrm{LXR} \alpha$ relative to the control treatment $(P<0.05$; Fig. $4(\mathrm{~d})$ and (e)). Furthermore, the transcriptional activity of LXRE-Luc was markedly induced in response to the 9-cis-RA treatment in 293T cells in a dose-dependent manner $(P<0 \cdot 01$; Fig. 4(f)).

\section{Liver $X$ receptor- $\alpha$ mediates retinoic acid-induced macrophage cholesterol efflux}

Compared with the cells treated with control siRNA, reduced cholesterol accumulation induced by 9-cis-RA was largely abolished in the presence of LXR $\alpha \operatorname{siRNA}(P<0 \cdot 01$; Fig. 5(a) and (b)). The knockdown efficiency of LXR $\alpha$ siRNA is shown in Fig. 5(c) and (d) $(P<0 \cdot 01)$. In addition, LXR $\alpha$ knockdown significantly suppressed 9-cis-RA-induced expression levels of both $A B C A 1$ and $A B C G 1$ ( $P<0.05$; Fig. 5(e) and (f)), leading to reduced cholesterol efflux $(P<0 \cdot 05$; Fig. 5(g) and (h)).

\section{Retinoic acid-induced macrophage cholesterol efflux is} farnesoid $X$ receptor and pregnane $X$ receptor independent

To explore the potential role of other heterodimeric partners of RXR, expression levels of LXR $\beta$, FXR and PXR were examined.
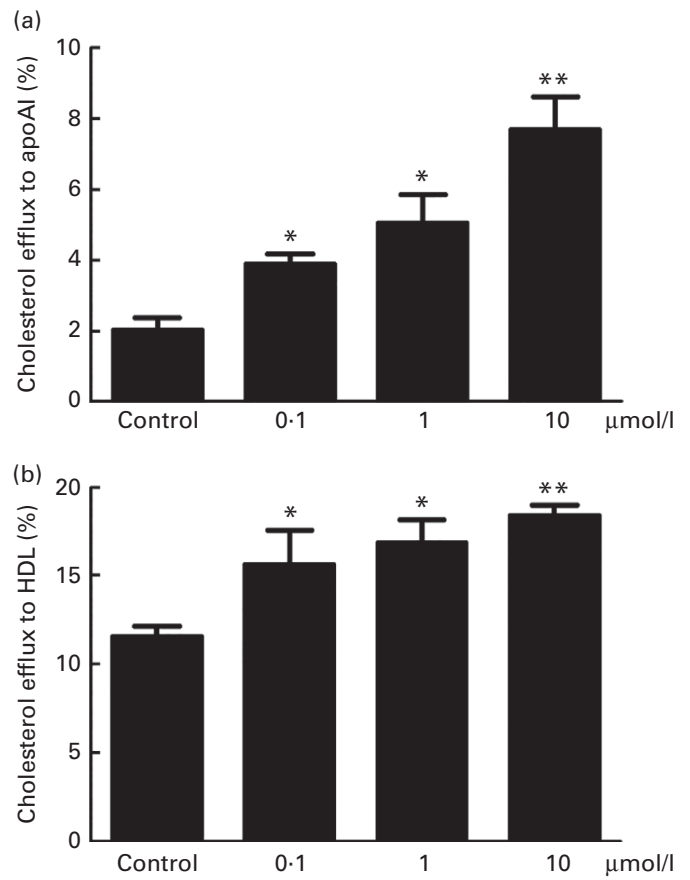

Fig. 3. Effects of 9-cis-retinoic acid (9-cis-RA) on NBD-cholesterol efflux from macrophages. J774A.1 macrophages were treated with vehicle or 9-cis-RA $(0 \cdot 1,1$ and $10 \mu \mathrm{mol} / \mathrm{l})$ for $24 \mathrm{~h}$, before equilibration with NBD-cholesterol for another $6 \mathrm{~h}$. Fluorescence in the cells and the medium was detected. Efflux of NBD-cholesterol to (a) apoAl and (b) HDL. Values are means, with their standard deviations represented by vertical bars. Mean value was significantly different from that of the control group: ${ }^{*} P<0.05$, ${ }^{* *} P<0.01$. 
(a)

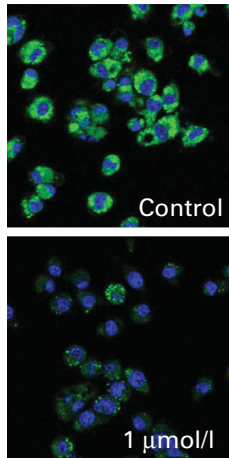

(c)
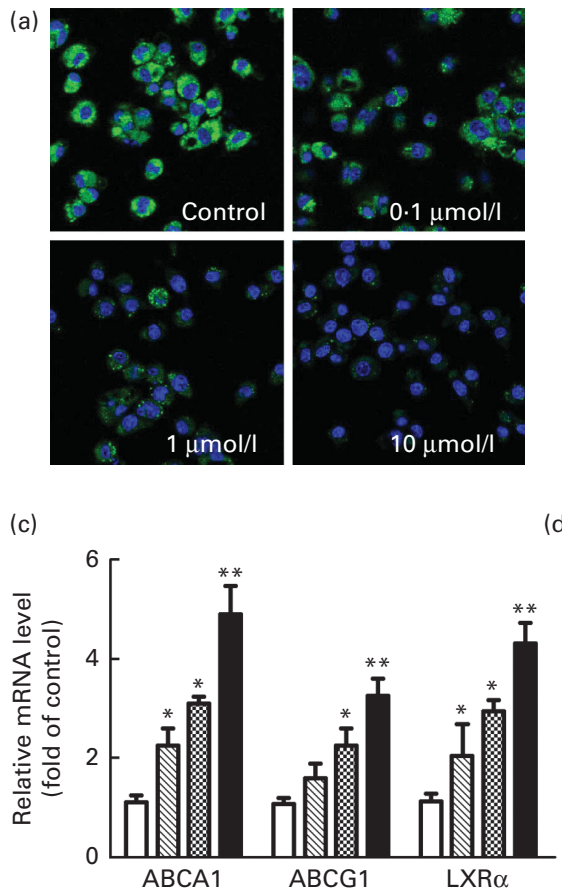

(b)

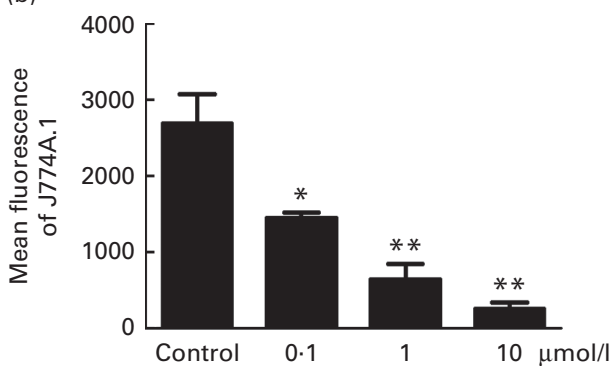

(d)

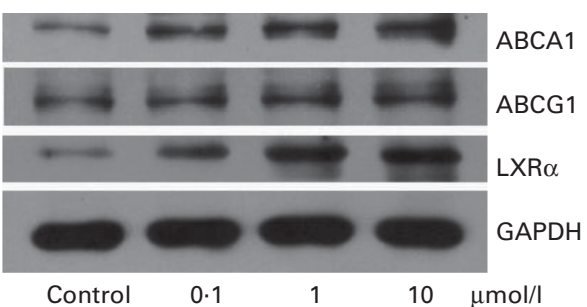

(e)

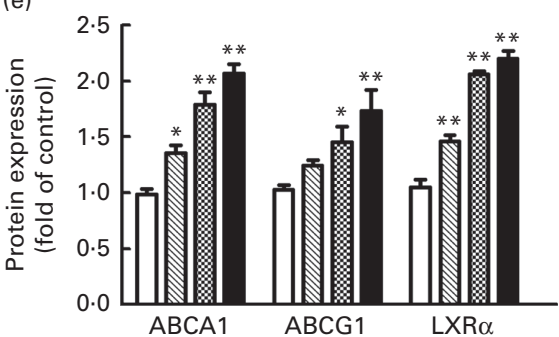

(f)

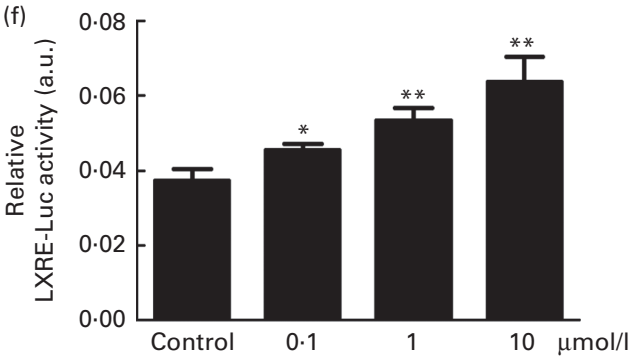

Fig. 4. Effects of 9-cis-retinoic acid (9-cis-RA) on the related gene expression levels of cholesterol transporters. J774A.1 cells were treated with vehicle or 9-cis-RA $(0.1,1$ and $10 \mu \mathrm{mol} / \mathrm{l})$ for $24 \mathrm{~h}$ before analysis. (a) Overlap images of BODIPY ${ }^{\circledR} 493 / 503$ and DAPI (4',6-diamidino-2-phenylindole). Magnification $400 \times$. (b) Mean fluorescence was calculated using ImageJ software. (c) The mRNA levels of ATP-binding cassette transporter A1 (ABCA1), ATP-binding cassette transporter $\mathrm{G} 1$ ( $A B C G 1)$ and liver $\mathrm{X}$ receptor- $\alpha(L X R \alpha)$ were determined by quantitative real-time PCR. (d) Protein expression levels of $A B C A 1, A B C G 1$ and $L X R \alpha$. (e) Quantification of ABCA1, ABCG1 and LXR $\alpha$ protein density normalised to the expression level of glyceraldehyde 3-phosphate dehydrogenase (GAPDH), and expressed as optical density relative to that of the control. (f) Liver X receptor response element (LXRE)-Luc activity in 293T cells. Values are means, with their standard deviations represented by vertical bars. Mean value was significantly different from that of the control group: ${ }^{\star} P<0.05$, ${ }^{\star \star} P<0.01$. $\square$, Control; $\mathbb{\nabla}, 0.1 \mu \mathrm{mol} / / ;$ 图, $1 \mu \mathrm{mol} / / ; \mathbf{\square}, 10 \mu \mathrm{mol} / /$. a.u., Arbitrary units. (A colour version of this figure can be found online at http://www.journals.cambridge.org/bjn).

Incubation with 9-cis-RA did not induce either the mRNA levels (Fig. 6(a)) or protein expression levels (Fig. 6(b) and (c)). Furthermore, the transcriptional activity of PXR was not influenced by 9-cis-RA (Fig. 6(d)). Although the transcriptional activity of FXR was stimulated by 9-cis-RA ( $P<0.05$; Fig. 6(d)), inhibition of FXR by a specific inhibitor exerted no obvious effect on the stimulation of ABCA1 and ABCG1 expression $(P<0.05$; Fig. 6(e) and (f)).

\section{Discussion}

In the present study, we have presented a novel finding of 9-cis-RA and its underlying mechanism for suppressing macrophage foam cell formation and atherosclerosis. First, we showed that 9-cis-RA prevented the acceleration of atherosclerosis in HFD-fed apoE ${ }^{-/-}$mice. MPM isolated from the RA group had higher expression levels of ABCA1/ABCG1 and lower cholesterol accumulation than those from the control group. The in vitro study further demonstrated that incubation with 9-cis-RA ameliorated oxidised LDL-induced lipid accumulation by dose-dependently inducing ABCA1/ABCG1 protein expression and cholesterol efflux via the up-regulation of LXR $\alpha$ transcriptional activity, and the effects were independent of FXR and PXR.

RA is an active metabolite of vitamin A that inhibits cell migration, regulates extrinsic coagulation, and promotes cell differentiation ${ }^{(26,27)}$. Its anti-tumour effects have been extensively studied for a long time ${ }^{(28,29)}$. Recently, studies have shown that RA limited restenosis after balloon angioplasty in a rabbit model ${ }^{(30)}$, suggesting a potential role in the prevention of atherosclerotic CVD. A previous study has shown that all-trans-RA ameliorated high-fat diet-induced atherosclerosis in rabbits by inhibiting platelet activation and inflammation ${ }^{(31)}$. However, the rabbit model was criticised 

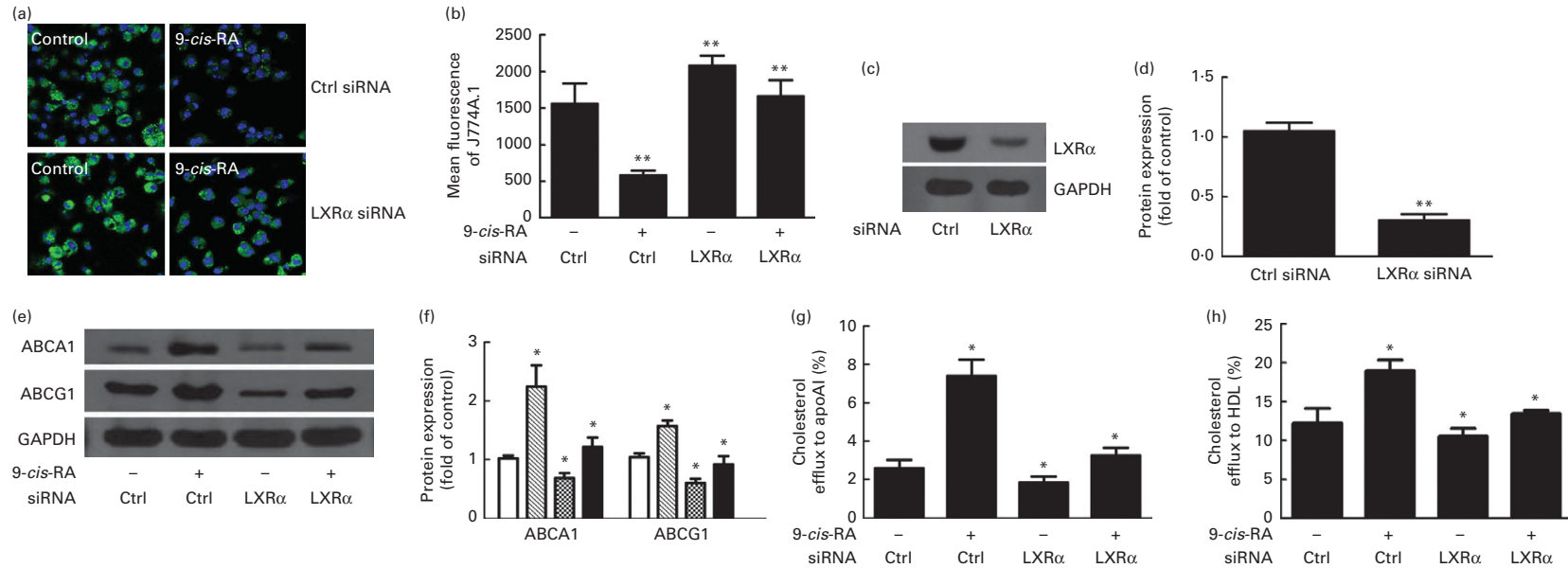

Fig. 5. Liver $X$ receptor- $\alpha(\operatorname{LXR} \alpha)$ mediates the induction of cholesterol efflux by 9-cis-retinoic acid (9-cis-RA). After transfection with LXR $\alpha$ small interfering RNA (siRNA) for $48 \mathrm{~h}$, J774A.1 cells were incubated with 9-cis-RA at a dose of $10 \mu \mathrm{mol} / / \mathrm{l}$ for another $24 \mathrm{~h}$ before analysis. (a) Overlap images of BODIPY ${ }^{\circledR} 493 / 503$ and DAPI (4',6-diamidino-2-phenylindole). Magnification 400x. (b) Mean fluorescence was calculated using ImageJ software. (c) Knockdown efficiency of LXR $\alpha$ siRNA. (d) Quantification of $L X R \alpha$ protein density normalised to the expression level of glyceraldehyde 3-phosphate dehydrogenase (GAPDH), and expressed as optical density relative to that of the control. (e) Protein expression levels of ATP-binding cassette transporter A1 (ABCA1) and ATP-binding cassette transporter G1 (ABCG1) were measured by Western blot. (f) Quantification of ABCA1 and ABCG1 protein density normalised to the expression level of GAPDH, and

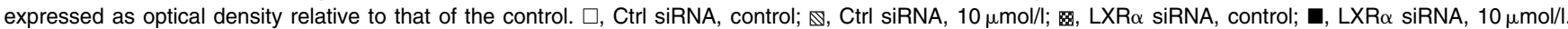
$(\mathrm{g}, \mathrm{h})$ After transfection with specific siRNA, cells were equilibrated with NBD-cholesterol. Efflux of NBD-cholesterol to $(\mathrm{g})$ apoAl and (h) HDL. Values are means, with their standard deviations represented by vertical bars. Mean value was significantly different from that of the control siRNA: ${ }^{*} P<0.05$, ${ }^{*} P<0.01$ (control siRNA/9-cis-RA v. LXR $\alpha$ siRNA/9-cis-RA). (A colour version of this figure can be found online at http://www.journals.cambridge.org/bjn).

for its use in atherosclerosis-nutritional studies because the majority of circulating cholesterol was transported in $\beta$-VLDL ${ }^{(32)}$, which does not mimic human lipoprotein metabolism and the development of aortic lesions. Studies have also indicated that RA reduced Chlamydia pneumoniae-accelerated foam cell lesion formation in hyperlipidaemic mice, but no exact mechanism has been elucidated ${ }^{(33)}$. Besides, retinoic acid-inducible gene-I $(R I G-I)$ has been reported to be involved in atherosclerosis by regulating the differentiation of macrophages ${ }^{(34)}$. Studies on retinoic acid receptor-related orphan receptor- $\alpha$ have also revealed that RA might be beneficial in the prevention of atherosclerosis via receptorrelated orphan receptor- $\alpha^{(35)}$. However, these two studies provided no direct link between RA and protection against (a)

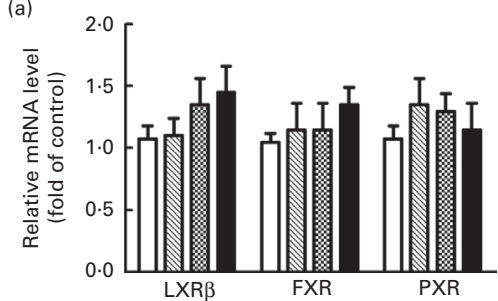

(d)

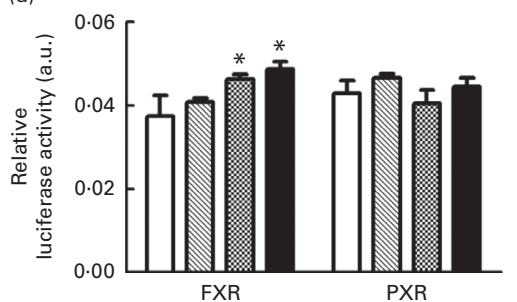

(b)

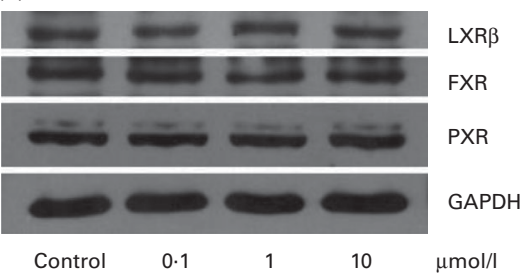

(e)

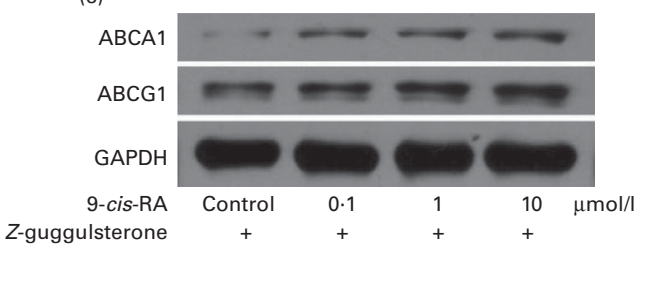

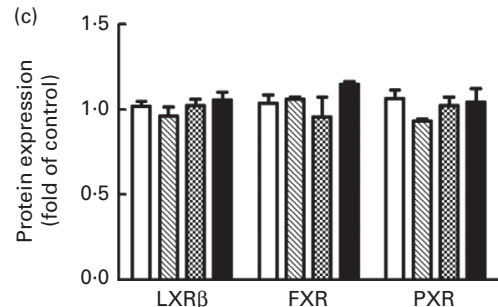

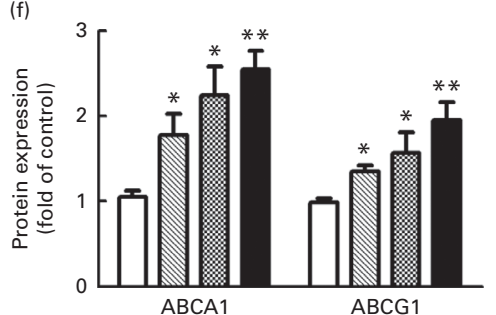

Fig. 6. Effects of 9-cis-retinoic acid (9-cis-RA) on the up-regulation of ATP-binding cassette transporter A1 (ABCA1) and ATP-binding cassette transporter G1 (ABCG1). The ABC transporters induced by 9-cis-RA were independent of farnesoid $X$ receptor (FXR) and pregnane $X$ receptor (PXR). (a-d) Cells were incubated with 9-cis-RA for $24 \mathrm{~h}$ before analysis. (a) mRNA levels of $L X R \beta$, FXR and PXR were determined by quantitative real-time PCR. (b) Protein expression levels of LXR $\beta$, FXR and PXR. (c) Quantification of LXR $\beta$, FXR and PXR protein density normalised to the expression level of glyceraldehyde 3-phosphate dehydrogenase (GAPDH), and expressed as optical density relative to that of the control. (d) Farnesoid $X$ receptor response element/pregnane $X$ receptor response element (FXRE/PXRE)-Luc activity in 293T cells. (e, f) J774A.1 macrophages were incubated with the combination of 9-cis-RA and Z-guggulsterone for $24 \mathrm{~h}$ before analysis. (e) Expression levels of ABCA1 and ABCG1. (f) Quantification of ABCA1 and ABCG1 protein density normalised to the expression level of GAPDH, and expressed as optical density relative to that of the control. Values are means, with their standard deviations represented by vertical bars. Mean

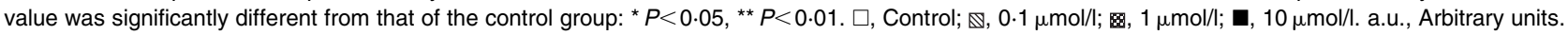


atherosclerosis. ApoE $\mathrm{E}^{-/-}$mice fed a Western-type diet represent a well-characterised animal model of the progression of atherosclerosis in humans ${ }^{(36)}$. Using this model, we observed that 9-cis-RA significantly ameliorated atherosclerotic lesion areas in the aortic sinus compared with the control treatment. To our knowledge, this is the first in vivo study demonstrating a direct anti-atherogenic effect of 9-cis-RA.

Atherogenic lipid abnormalities, characterised by low HDL-cholesterol levels and high TAG levels together with the presence of small, dense, atherogenic LDL particles, contribute substantially to the development of atherosclerosis ${ }^{(37,38)}$. In the present study, apoE ${ }^{-/-}$mice treated with RA exhibited lower serum levels of total cholesterol $(35.9 \%$ reduction) and LDL-cholesterol (26.1\% reduction), but higher levels of HDL-cholesterol $(32 \cdot 1 \%$ increase $)$ compared with those treated with vehicle (control). Elevated serum HDL-cholesterol levels were correlated with a reduced risk of atherosclerosis $^{(39)}$, on the basis that HDL play an important role in transporting cholesterol or cholesterol ester from peripheral tissues to the liver where cholesterol is metabolised and transformed into bile acids. The LDL receptor ( $L d l r$ ) gene, a direct target gene of LXR, has been proved to play a key role in mediating the endocytic uptake of LDL-cholesterol in the liver ${ }^{(40)}$. In the present study, we showed that the 9-cis-RA treatment significantly induced the transcriptional activity of LXRE, which might induce the expression levels of hepatic LDLR and lead to a decrease in serum total and LDL-cholesterol concentrations. This pathway represents one critically important mechanism by which 9-cis-RA reduces cholesterol concentrations in both blood and peripheral tissues, thus protecting against atherosclerotic plaque formation in apoE $^{-/-}$mice.

ABCA1 and ABCG1 are the members of the ATP-binding cassette transporter family (ABC transporters), and have been shown to regulate cellular lipid metabolism ${ }^{(8-10)}$. Studies using gene-manipulated mice have demonstrated that foam cell accumulation and atherosclerotic lesions were significantly promoted in individual transporter-deficient mice ${ }^{(41,42)}$. Therefore, we examined the regulatory role of 9-cis-RA, and showed that protein expression levels of ABCA1 and ABCG1 were higher in the MPM of $\mathrm{apoE}^{-/-}$mice treated with 9-cis-RA compared with those treated with vehicle. Thus, our findings indicate that the induction of ABCA1 and ABCG1 by 9-cis-RA may contribute to the protection of atherogenesis by affecting cholesterol efflux during the transformation of foam cells. Moreover, we demonstrated that RA treatment mitigated lipid accumulation in cholesterol-preloaded macrophages, as evidenced by lower BODIPY ${ }^{\circledR} 493 / 503$ immunofluorescent staining in MPM from the RA group compared with the control group. Retinoids have been implicated as potent inducers of genes related to human lipid metabolism in various macrophage cell lines ${ }^{(43)}$. Retinoic acid receptor activators (all-trans-RA and 4-[E-2-5,6,7,8-tetrahydro-5,5,8,8-tetramethyl2-naphthalenyl-1-propenyl] benzoic acid (TTNPB)) were found to increase the mRNA and protein expression levels of ABCA1 in macrophages ${ }^{(44)}$. However, these studies failed to specify the key role of LXR, FXR and PXR in the mediation of 9-cis-RA-induced $A B C A 1$ and $A B C G 1$ expression and reduced foam cell formation, a crucial early stage in atherogenesis. In accordance with previous studies that demonstrated a stimulatory role of LXR $\alpha$ and/or LXR $\beta$ and RXR in the activation of $\mathrm{ABCA} 1^{(45,46)}$, our results revealed that 9-cisRA increased the transcriptional activity of LXRE in the promoter of ABCA1/ABCG1. Consistent with the observation that in macrophages, LXR regulates cholesterol efflux by the $L X R \alpha$ gene rather than by $L X R \beta^{(47,48)}$, we observed no significant change in $L X R \beta$ expression at both mRNA and protein levels, and conclude that increased LXR activity was mainly attributable to LXR $\alpha$. In addition, the mRNA and protein expression levels of LXR $\beta$, FXR and PXR were not obviously increased by 9-cis-RA. Although the 9-cis-RA treatment had a slight effect on the transcriptional activity of FXR, the protein expression levels of ABCA1 and ABCG1 were still increased dose-dependently when treated with a specific FXR inhibitor, indicating that FXR may not play an important role in the 9-cisRA-induced up-regulation of ABCA1 and ABCG1 in macrophages. 9-cis-RA did not have an effect on the transcriptional activity of PXR. Collectively, these results excluded the role of FXR and PXR, another two potential heterodimeric partners of RXR modulating systematic lipid metabolism, in the 9-cis-RAinduced up-regulation of ABCA1 and ABCG1. All these data imply that the 9-cis-RA-induced up-regulation of $A B C A 1$ and $A B C G 1$ and cholesterol efflux are only dependent on the activation of $\operatorname{LXR} \alpha$.

Taken together, the present study is the first to reveal that 9-cis-RA significantly ameliorates HFD-induced atherosclerosis in vivo, and presents a detailed molecular mechanism by which 9-cis-RA protects against HFD-induced atherosclerosis through inducing cholesterol efflux from macrophages via the LXR $\alpha$-dependent up-regulation of ABCA1 and ABCG1.

\section{Acknowledgements}

The present study was supported by grants from the National Natural Science Foundation of China (no. 81172663, 81072301) and the National Natural Science Foundation from Guangdong Province (no. S2012020011104).

The authors' contributions are as follows: W. Z. and J. L. conducted the research; H. C., J. W. and Y. L. analysed the data; M. X. designed the experiments and prepared the manuscript. All authors read and approved the final manuscript

None of the authors has any conflict of interest to declare.

\section{References}

1. Glass CK \& Witztum JL (2001) Atherosclerosis: the road ahead. Cell 104, 503-516.

2. Lusis AJ (2000) Atherosclerosis. Nature 407, 233-241.

3. Li AC \& Glass CK (2002) The macrophage foam cell as a target for therapeutic intervention. Nat Med 8, 1235-1242.

4. Ouimet M \& Marcel YL (2012) Regulation of lipid droplet cholesterol efflux from macrophage foam cells. Arterioscler Thromb Vasc Biol 32, 575-581.

5. Phillips MC (2014) Molecular mechanisms of cellular cholesterol efflux. J Biol Chem 289, 20420-20429. 
6. Kennedy MA, Barrera GC, Nakamura K, et al. (2005) ABCG1 has a critical role in mediating cholesterol efflux to HDL and preventing cellular lipid accumulation. Cell Metab 1, 121-131.

7. Wang N, Silver DL, Thiele C, et al. (2001) ATP-binding cassette transporter A1 (ABCA1) functions as a cholesterol efflux regulatory protein. J Biol Chem 276, 23742-23747.

8. Gelissen IC, Harris M, Rye KA, et al. (2006) ABCA1 and ABCG1 synergize to mediate cholesterol export to apoA-I. Arterioscler Thromb Vasc Biol 26, 534-540.

9. Jessup W, Gelissen IC, Gaus K, et al. (2006) Roles of ATP binding cassette transporters A1 and G1, scavenger receptor BI and membrane lipid domains in cholesterol export from macrophages. Curr Opin Lipidol 17, 247-257.

10. Tall AR, Yvan-Charvet L, Terasaka N, et al. (2008) HDL, ABC transporters, and cholesterol efflux: implications for the treatment of atherosclerosis. Cell Metab 7, 365-375.

11. Venkateswaran A, Laffitte BA, Joseph SB, et al. (2000) Control of cellular cholesterol efflux by the nuclear oxysterol receptor LXR $\alpha$. Proc Natl Acad Sci U S A 97, 12097-12102.

12. Sabol SL, Brewer HB \& Santamarina-Fojo S (2005) The human $A B C G 1$ gene: identification of LXR response elements that modulate expression in macrophages and liver. J Lipid Res 46, 2151-2167.

13. Zile MH (2001) Function of vitamin A in vertebrate development. J Nutr 131, 705-708.

14. Ross SA, McCaffery PJ, Drager UC, et al. (2000) Retinoids in embryonal development. Physiol Rev 80, 1021-1054.

15. Petkovich PM (2001) Retinoic acid metabolism. J Am Acad Dermatol 45, 136-142.

16. Blomhoff R \& Blomhoff HK (2006) Overview of retinoid metabolism and function. J Neurobiol 66, 606-630.

17. Shulman AI \& Mangelsdorf DJ (2005) Retinoid X receptor heterodimers in the metabolic syndrome. $N$ Engl $\mathrm{J}$ Med 353, 604-615.

18. Heyman RA, Mangelsdorf DJ, Dyck JA, et al. (1992) Retinoic acid is a high affinity ligand for the retinoid $\mathrm{X}$ receptor. Cell 68, 397-406.

19. Zhang Y, Beyer TP, Bramlett KS, et al. (2002) Liver X receptor and retinoic $\mathrm{X}$ receptor mediated $\mathrm{ABCA} 1$ regulation and cholesterol efflux in macrophage cells-messenger RNA measured by branched DNA technology. Mol Genet Metab 77, 150-158

20. Ayaori M, Yakushiji E, Ogura M, et al. (2012) Retinoic acid receptor agonists regulate expression of ATP-binding cassette transporter G1 in macrophages. Biochim Biophys Acta 1821, 561-572.

21. Koldamova RP, Lefterov IM, Ikonomovic MD, et al. (2003) $22 R$-Hydroxycholesterol and 9-cis-retinoic acid induce ATPbinding cassette transporter A1 expression and cholesterol efflux in brain cells and decrease amyloid $\beta$ secretion. J Biol Chem 278, 13244-13256.

22. Wang Y, Zhang Y, Wang X, et al. (2012) Supplementation with cyanidin-3-O- $\beta$-glucoside protects against hypercholesterolemia-mediated endothelial dysfunction and attenuates atherosclerosis in apolipoprotein E-deficient mice. $J$ Nutr 142, 1033-1037.

23. Xia M, Hou M, Zhu $\mathrm{H}$, et al. (2005) Anthocyanins induce cholesterol efflux from mouse peritoneal macrophages: the role of the peroxisome proliferator-activated receptor $\gamma$-liver X receptor $\alpha$-ABCA1 pathway. J Biol Chem 280, 36792-36801.

24. Lu KY, Ching LC, Su KH, et al. (2010) Erythropoietin suppresses the formation of macrophage foam cells: role of liver X receptor $\alpha$. Circulation 121, 1828-1837.
25. Singh R, Kaushik S, Wang Y, et al. (2009) Autophagy regulates lipid metabolism. Nature 458, 1131-1135.

26. Ou H, Haendeler J, Aebly MR, et al. (2000) Retinoic acidinduced tissue transglutaminase and apoptosis in vascular smooth muscle cells. Circ Res 87, 881-887.

27. Axel DI, Frigge A, Dittmann J, et al. (2001) All-trans retinoic acid regulates proliferation, migration, differentiation, and extracellular matrix turnover of human arterial smooth muscle cells. Cardiovasc Res 49, 851-862.

28. Tallman MS, Andersen JW, Schiffer CA, et al. (2002) All-trans retinoic acid in acute promyelocytic leukemia: long-term outcome and prognostic factor analysis from the North American Intergroup protocol. Blood 100, 4298-4302.

29. Ponthan F, Kogner P, Bjellerup P, et al. (2001) Bioavailability and dose-dependent anti-tumor effects of 9-cis retinoic acid on human neuroblastoma xenografts in rat. Br J Cancer $\mathbf{8 5}$, 2004-2009.

30. Herdeg C, Oberhoff M, Baumbach A, et al. (2003) Effects of local all-trans-retinoic acid delivery on experimental atherosclerosis in the rabbit carotid artery. Cardiovasc Res 57, $544-553$

31. Zhou B, Pan Y, Hu Z, et al. (2012) All-trans-retinoic acid ameliorated high fat diet-induced atherosclerosis in rabbits by inhibiting platelet activation and inflammation. J Biomed Biotechnol 2012, 259693.

32. Mahley RW (1978) Alterations in plasma lipoproteins induced by cholesterol feeding in animals including man. In Disturbance in Lipid and Lipoprotein Metabolism, pp. 181-197 [JM Dietschy, AM Gotto and JA Ontko, editors]. Bethesda, MD: American Physiological society.

33. Jiang SJ, Campbell LA, Berry MW, et al. (2008) Retinoic acid prevents Chlamydia pneumoniae-induced foam cell development in a mouse model of atherosclerosis. Microbes Infect 10, 1393-1397.

34. Imaizumi T, Yagihashi N, Kubota K, et al. (2007) Expression of retinoic acid-inducible gene-I $(R I G-I)$ in macrophages: possible involvement of $R I G-I$ in atherosclerosis. $J$ Atheroscler Thromb 14, 51-55.

35. Jakel H, Fruchart-Najib J \& Fruchart JC (2006) Retinoic acid receptor-related orphan receptor $\alpha$ as a therapeutic target in the treatment of dyslipidemia and atherosclerosis. Drug News Perspect 19, 91-97.

36. Nakashima Y, Plump AS, Raines EW, et al. (1994) ApoE deficient mice develop lesions of all phases of atherosclerosis throughout the arterial tree. J Arterioscler Thromb 14, 133-140.

37. Hoogeveen RC, Gaubatz JW, Sun W, et al. (2014) Small dense low-density lipoprotein-cholesterol concentrations predict risk for coronary heart disease: the Atherosclerosis Risk In Communities (ARIC) study. Arterioscler Thromb Vasc Biol 34, 1069-1077.

38. Carmena R, Duriez P \& Fruchart JC (2004) Atherogenic lipoprotein particles in atherosclerosis. Circulation 109, III2-III7.

39. Stein O \& Stein Y (1999) Atheroprotective mechanisms of HDL. Atherosclerosis 144, 285-301.

40. Ishimoto K, Tachibana K, Sumitomo M, et al. (2006) Identification of human low-density lipoprotein receptor as a novel target gene regulated by liver $\mathrm{X}$ receptor $\alpha$. FEBS Lett 580, 4929-4933.

41. Yvan-Charvet L, Ranalletta M, Wang N, et al. (2007) Combined deficiency of ABCA1 and ABCG1 promotes foam cell accumulation and accelerated atherosclerosis in mice. $J$ Clin Invest 117, 3900-3908.

42. Wang X, Collins HL, Ranalletta M, et al. (2007) Macrophage ABCA1 and ABCG1, but not SR-BI, promote macrophage 
reverse cholesterol transport in vivo. I Clin Invest $\mathbf{1 1 7}$ 2216-2224.

43. Langmann T, Liebisch G, Moehle C, et al. (2005) Gene expression profiling identifies retinoids as potent inducers of macrophage lipid efflux. Biochim Biophys Acta 1740, 155-161.

44. Costet P, Lalanne F, Gerbod-Giannone MC, et al. (2003) Retinoic acid receptor-mediated induction of ABCA1 in macrophages. Mol Cell Biol 23, 7756-7766.

45. Zhang Y, Beyer TP, Bramlett KS, et al. (2002) Liver X receptor and retinoic $\mathrm{X}$ receptor mediated ABCA1 regulation and cholesterol efflux in macrophage cells-messenger RNA measured by branched DNA technology. Mol Genet Metab 77, 150-158.
46. Kritharides L, Christian A, Stoudt G, et al. (1998) Cholesterol metabolism and efflux in human THP-1 macrophages. Arterioscler Thromb Vasc Biol 18, 1589-1599.

47. Whitney KD, Watson MA, Goodwin B, et al. (2001) Liver $\mathrm{X}$ receptor (LXR) regulation of the $L X R \alpha$ gene in human macrophages. J Biol Chem 276, 43509-43515.

48. Ma AZ, Song ZY \& Zhang Q (2014) Cholesterol efflux is LXR $\alpha$ isoform-dependent in human macrophages. BMC Cardiovasc Disord 14, 80.

49. Reeves PG, Nielsen FH \& Fahey GC Jr (1993) AIN-93 purified diets for laboratory rodents: final report of the American Institute of Nutrition ad hoc writing committee on the reformulation of the AIN-76A rodent diet. $J$ Nutr 123, 1939-1951. 